\title{
Pattern Recognition Via Synchronization in Phase-Locked Loop Neural Networks
}

\author{
Frank C. Hoppensteadt, Member, IEEE, and Eugene M. Izhikevich
}

\begin{abstract}
We propose a novel architecture of an oscillatory neural network that consists of phase-locked loop (PLL) circuits. It stores and retrieves complex oscillatory patterns as synchronized states with appropriate phase relations between neurons.
\end{abstract}

Index Terms-Brain rhythms, oscillatory associative memory, temporal pattern recognition, voltage-controlled oscillators (VCO's).

\section{INTRODUCTION}

$\mathbf{O}$ SCILLATIONS are ubiquitous in nature and, in particular, in neuron and brain dynamics. There is a mounting evidence that rhythmic activity and synchronization of neuronal firing play a profound role in the information processing in many brain systems, including the olfactory bulb, hippocampus, and thalamo-cortical system; see [4] for a review. However, much of the artificial neural network research still focuses on nonoscillatory sigmoidal neurons whose dynamics correspond to the mean firing rates. The precise timing of neuronal firing is usually neglected.

To understand possible neurocomputational properties of oscillatory neural networks we consider an extreme case when each neuron exhibits periodic activity. Such networks can be described by phase models (see [9, ch. 9] for rigorous introduction to the theory) and take into account precise timing of spikes. In particular, we have proven an analogue of Cohen-Grossberg convergence theorem [3], but for oscillatory neural networks (see [9, Th. 9.15] and [1]). It shows that oscillatory neural networks can have all the neurocomputational properties of standard Hopfield networks, with a key exception: Memorized patterns are not equilibria, but synchronized oscillatory states in which neurons fire periodically with certain relation between their phases (firing times). This approach is in the spirit of that developed in [1], [2], [9], and [16].

Interaction between such neurons occurs via modulation of their phases (PM encoding), and it depends crucially on the resonant relations between their frequencies. Such a dependence is the key issue of the frequency-modulated (FM) interaction theory ([6]-[9], [13]-[15]), which shows that oscillatory networks can have other neurocomputational properties far exceeding those of Hopfield-Grossberg networks.

In this paper, we suggest an implementation of an oscillatory neural network using phase-locked loops (PLL's) (see Fig. 1).

Manuscript received July 19, 1999; revised February 11, 2000.

F. C. Hoppensteadt is with the Center for Systems Science and Engineering, Arizona State University, Tempe, AZ 85287-7606 USA.

E. M. Izhikevich is with The Neurosciences Institute, La Jolla, CA 92121 USA.

Publisher Item Identifier S 1045-9227(00)04298-3.
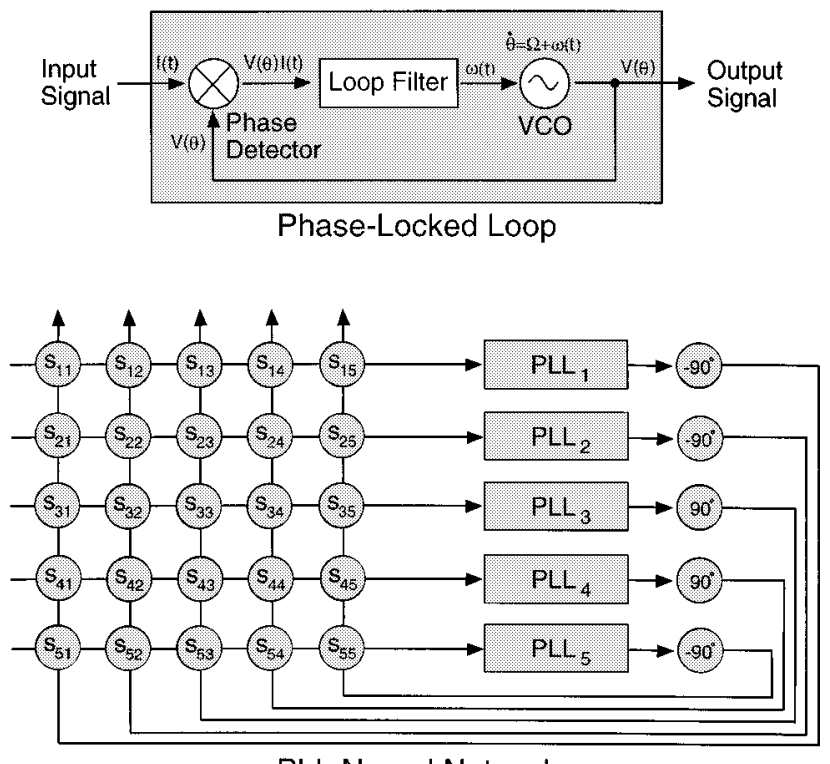

PLL Neural Network

Fig. 1. Conceptual architecture of the PLL neural network described by (1). Notice that the VCO outputs are phase-shifted by $-90^{\circ}(-\pi / 2)$, so that each PLL receives input signal $I_{i}=\sum_{j=1}^{n} s_{i j} V\left(\vartheta_{j}-\pi / 2\right)$.

Its major advantage is that PLL circuit technology is well developed and understood [5], [6], [12].

\section{Phase-Locked Loop Neural Network}

In this section, we consider a dynamical system

$$
\dot{\vartheta}_{i}=\Omega+V\left(\vartheta_{i}\right) \sum_{j=1}^{n} s_{i j} V\left(\vartheta_{j}-\pi / 2\right)
$$

which describes the dynamics of the PLL neural network depicted in Fig. 1. Here $\vartheta_{i}$ is the phase of the voltage-controlled oscillator (VCO) embedded in the $i$ th PLL, $\Omega \gg 1$ is its natural frequency (megahertz), $s_{i j}$ are connection coefficients, and $V$ is a $2 \pi$-periodic output waveform function, which is assumed to satisfy the following "odd-even" condition.

- (Odd) Waveform $V(\vartheta)$ is an odd function; that is

$$
V(-\vartheta)=-V(\vartheta)
$$

for all $\vartheta$.

- (Even) Phase-shifted waveform $V(\vartheta-\pi / 2)$ is an even function; that is

$$
V\left(-\vartheta-\frac{\pi}{2}\right)=V(\vartheta-\pi / 2)
$$

for all $\vartheta$. 

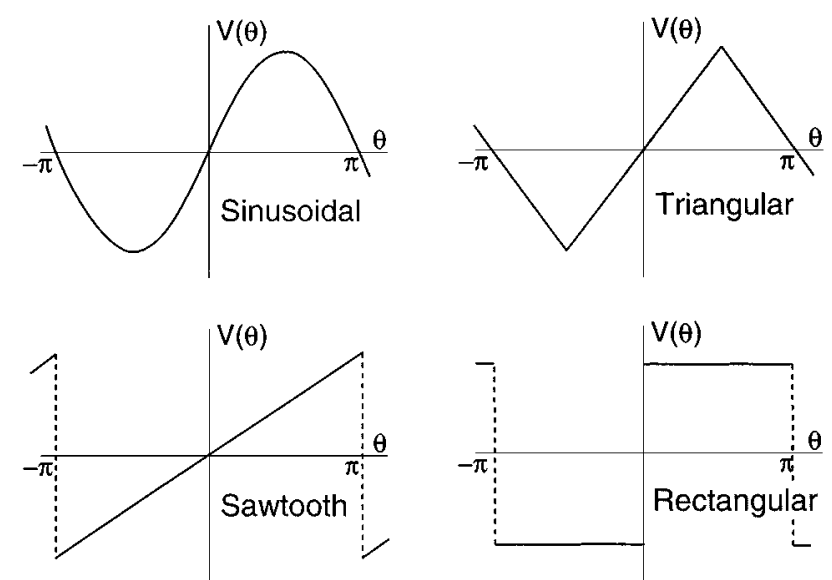

Fig. 2. Typical waveforms found in commercially available PLL's.

Notice that three out of four standard waveforms found in commercially available PLL's and depicted in Fig. 2 satisfy the "odd-even" condition, at least after a phase shift. The "sawtooth" waveform does not satisfy the "even" part of the condition.

The key mathematical result of this paper is the following theorem.

Theorem 1 (Synchronization Theorem for PLL Neural Networks): Consider the PLL neural network (1) depicted in Fig. 1 with an arbitrary waveform function $V$ satisfying the "odd-even" condition above. If

$$
s_{i j}=s_{j i}, \quad \text { for all } i \text { and } j
$$

then the network always converges to an oscillatory phase-locked pattern; that is, the neurons oscillate with equal frequencies and constant, but not necessarily zero phase relations.

There could be many such phase-locked patterns corresponding to many memorized images. Thus, the key difference between the Hopfield-Grossberg and the PLL neural network is that memorized images correspond to equilibrium (point) attractors in the former and limit cycle attractors in the latter. Pattern recognition by the PLL neural network consists in convergence to the corresponding limit cycle attractor, which results in synchronization of the network activity with an appropriate phase relation between neurons, as in Fig. 5. In the subsequent sections, we illustrate this issue and show how the PLL neural network memorizes and retrieves complex oscillatory patterns.

Proof: Let $\varphi_{i}$ be the phase deviation of the $i$ th oscillator from the natural phase $\Omega t$; that is

$$
\vartheta_{i}(t)=\Omega t+\varphi_{i}
$$

We want to show that the vector of phase deviations, $\varphi=\left(\varphi_{1}, \cdots, \varphi_{n}\right)$, converges to an equilibrium, say $\varphi^{\star}$. This corresponds to a synchronization of the network activity with the pattern of phase relations $\varphi^{\star}$. The proof is a standard application of averaging and Fourier analysis, and it consists of three steps [5], [9].
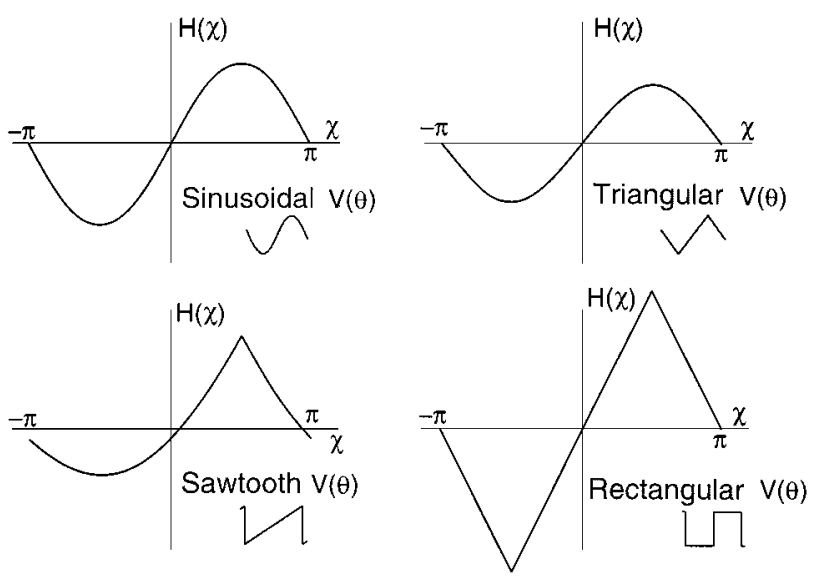

Fig. 3. Functions $H(\chi)$ for various waveforms $V(\vartheta)$ from Fig. 2.

Step I (averaging): We can rewrite the system (1) in the phase deviation form

$$
\dot{\varphi}_{i}=V\left(\Omega t+\varphi_{i}\right) \sum_{j=1}^{n} s_{i j} V\left(\Omega t+\varphi_{j}-\pi / 2\right) .
$$

Since $\Omega \gg 1$, we can average it (this is the purpose of the loop filter in the PLL in Fig. 1) to obtain

$$
\dot{\varphi}_{i}=\sum_{j=1}^{n} s_{i j} H\left(\varphi_{j}-\varphi_{i}\right)
$$

plus higher order terms, where

$H\left(\varphi_{j}-\varphi_{i}\right)=\lim _{T \rightarrow \infty} \frac{1}{T} \int_{0}^{T} V\left(\Omega t+\varphi_{i}\right) V\left(\Omega t+\varphi_{j}-\pi / 2\right) d t$ is the "averaged" connection function.

Step II (Fourier expansions): Let us show that

$$
H(\chi)=\sum_{m=1,3,5 \ldots}^{\infty} \frac{a_{m}^{2}}{2}(-1)^{m-1 / 2} \sin m \chi
$$

where $a_{m}$ are Fourier coefficients of the waveform function $V(\vartheta)$. In particular, $H(\chi)=1 / 2 \sin \chi$ when $V(\vartheta)=\sin \vartheta$ see also Fig. 3. Indeed, we can expand the $2 \pi$-periodic function $V(\vartheta)$ into its Fourier series, which has the form

$$
V(\vartheta)=\sum_{m=1,3,5 \cdots}^{\infty} a_{m} \sin m \vartheta
$$

due to the "odd-even" condition above. One can check that

$$
V(\vartheta-\pi / 2)=\sum_{m=1,3,5 \ldots}^{\infty}(-1)^{m+1 / 2} a_{m} \cos m \vartheta
$$

The product $V\left(\vartheta_{i}\right) V\left(\vartheta_{j}-\pi / 2\right)$ is given by

$$
\sum_{m, k=1,3,5 \ldots}^{\infty} a_{m} a_{k}(-1)^{k+1 / 2} \sin m \vartheta_{i} \cos k \vartheta_{j}
$$

which we rewrite in the form

$$
\begin{aligned}
& \sum_{m, k=1,3,5 \ldots}^{\infty} \frac{a_{m} a_{k}}{2}(-1)^{k+1 / 2}\left(\sin \left(m \vartheta_{i}+k \vartheta_{j}\right)\right. \\
& \left.\quad+\sin \left(m \vartheta_{i}-k \vartheta_{j}\right)\right) .
\end{aligned}
$$

The only low-frequency modes, which can survive the averaging, are those given by $\sin \left(m \vartheta_{i}-k \vartheta_{j}\right)$ when $m=k$, which results in (4). 


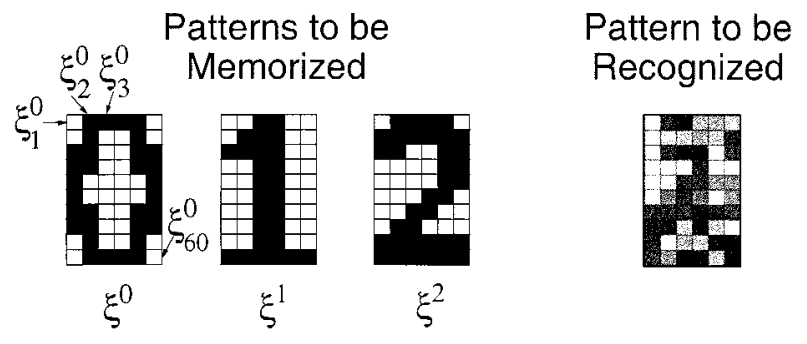

Fig. 4. Key patterns to be memorized. Neurons having similar phases are depicted using similar colors.

Step III (convergence): Now we use the condition $s_{i j}=s_{j i}$ to prove that phase deviation model (3) is a gradient system with the potential function

$$
U(\varphi)=\frac{1}{2} \sum_{i=1}^{n} \sum_{j=1}^{n} s_{i j} R\left(\varphi_{j}-\varphi_{i}\right)
$$

where $R$ is an antiderivative of $H$; i.e., $R^{\prime}=H$. The former is an even function because the latter is odd. One can easily check that $\dot{\varphi}_{i}=-\partial U / \partial \varphi_{i}$ for all $i$.

We see that if the matrix of synaptic connections is symmetric, then the PLL network synchronizes with a certain pattern of phase relations, which is determined by the attractors of (3). In the next section, we use $V(\vartheta)=\sin \vartheta$ to illustrate the neurocomputational properties of the PLL networks. Thus, we consider a system

$$
\dot{\vartheta}_{i}=\Omega-\sin \vartheta_{i} \sum_{j=1}^{n} s_{i j} \cos \vartheta_{j} .
$$

Assuming $\vartheta_{i}(t)=\Omega t+\varphi_{i}$, the corresponding phase deviation model (3) has a simple form

$$
\dot{\varphi}_{i}=\frac{1}{2} \sum_{j=1}^{n} s_{i j} \sin \left(\varphi_{j}-\varphi_{i}\right) .
$$

Other choices of the waveform $V(\vartheta)$ result in different $H$ (see Fig. 3). Whether they can provide any advantages remains an open question.

\section{ILLUSTRATION}

Suppose we are given a set of key vectors (patterns) to be memorized

$$
\xi^{k}=\left(\xi_{1}^{k}, \xi_{2}^{k}, \cdots, \xi_{n}^{k}\right), \quad \xi_{i}^{k}= \pm 1, \quad k=0, \cdots, p
$$

e.g., those in Fig. 4, where $\xi_{i}^{k}=\xi_{j}^{k}$ means that the $i$ th and the $j$ th oscillators are in-phase $\left(\varphi_{i}=\varphi_{j}\right)$ and $\xi_{i}^{k}=-\xi_{j}^{k}$ means they are antiphase $\left(\varphi_{i}=\varphi_{j}+\pi\right)$. First, notice that the problem of negative images does not exist in oscillatory neural networks, since both $\xi^{k}$ and $-\xi^{k}$ result in the same phase relations. A Hebbian learning rule of the form

$$
s_{i j}=\frac{1}{n} \sum_{k=0}^{p} \xi_{i}^{k} \xi_{j}^{k}
$$

is the simplest one (but not the best) among many possible learning algorithms. It produces symmetric connections $s_{i j}$ so that the Synchronization Theorem for PLL Neural Networks is applicable.
We use the learning rule to train the network with three images " 0 ," "1," and "2" depicted in Fig. 4. When the initial phase distribution corresponds to a distorted image " 1 ," the oscillators lock to each other with an appropriate phase relation (in-phase or antiphase), which is referred to as associative memory recall (see top of Fig. 5). We also plot two VCO outputs, $V\left(\vartheta_{1}\right)$ and $V\left(\vartheta_{2}\right)$, and their phase deviations, $\varphi_{1}$ and $\varphi_{2}$. As one expects, the oscillators approach a nearly in-phase-locked state during the recall.

To extract the relative phase pattern we need to remove the high frequency oscillatory component from the recognized image. For this we note that the product

$$
V\left(\vartheta_{1}\right) V\left(\vartheta_{i}\right) \rightarrow \cos \left(\vartheta_{i}-\vartheta_{1}\right)=\cos \left(\varphi_{i}-\varphi_{1}\right)
$$

after low-pass filtering. We plot the quantities $V\left(\vartheta_{1}\right) V\left(\vartheta_{i}\right), i=$ $1, \cdots, n$ at the bottom of Fig. 5. One can clearly see the relative phase pattern.

\section{COMPlex Oscillatory PatTerns}

A generalization of the PLL neural network (1) is

$$
\dot{\vartheta}_{i}=\Omega+V\left(\vartheta_{i}\right) \sum_{j=1}^{n} s_{i j} V\left(\vartheta_{j}-\pi / 2+\psi_{i j}\right)
$$

where $\psi_{i j}$ are natural phase shifts between neurons, also referred to as being phases (angles) of synaptic connections.

The Synchronization Theorem for PLL Neural Networks would need an additional condition

$$
\psi_{i j}=-\psi_{j i}, \quad \text { for all } i \text { and } j
$$

which combined with (2) can be expressed compactly as

$$
c_{i j}=\bar{c}_{j i}, \quad \text { for all } i \text { and } j
$$

where $c_{i j}=s_{i j} e^{\mathrm{i} \psi_{i j}}$ is a complex synaptic coefficient and $\bar{c}$ means complex conjugation. The Theorem's proof is unchanged, except that system (3) then has the form

$$
\dot{\varphi}_{i}=\sum_{j=1}^{n} s_{i j} H\left(\varphi_{j}+\psi_{i j}-\varphi_{i}\right)
$$

and the potential function (5) is

$$
U(\varphi)=\frac{1}{2} \sum_{i=1}^{n} \sum_{j=1}^{n} s_{i j} R\left(\varphi_{j}+\psi_{i j}-\varphi_{i}\right) .
$$

Such a network can memorize more complicated oscillatory patterns than mere in-phase/antiphase relations defined by the real key vectors (6). Indeed, we can consider complex vectors $\xi^{k}$ so that the desired phase shift between the $i$ th and the $j$ th neuron is the argument (angle) difference between the complex numbers $\xi_{i}^{k}$ and $\xi_{j}^{k}$. For example, the merry-go-around state can be represented as

$$
\xi=\left(1, e^{\mathrm{i} / n}, e^{\mathrm{i} 2 / n}, e^{\mathrm{i} 3 / n}, \cdots, e^{\mathrm{i}(n-1) / n}\right) \in \mathbb{C}^{n} .
$$

To memorize such phase patterns we can employ the complex Hebbian learning rule [1], [9]-[11]

$$
c_{i j}=\frac{1}{n} \sum_{k=0}^{p} \xi_{i}^{k} \bar{\xi}_{j}^{k}
$$

which modifies both the strength and the phase of the synaptic connections. 

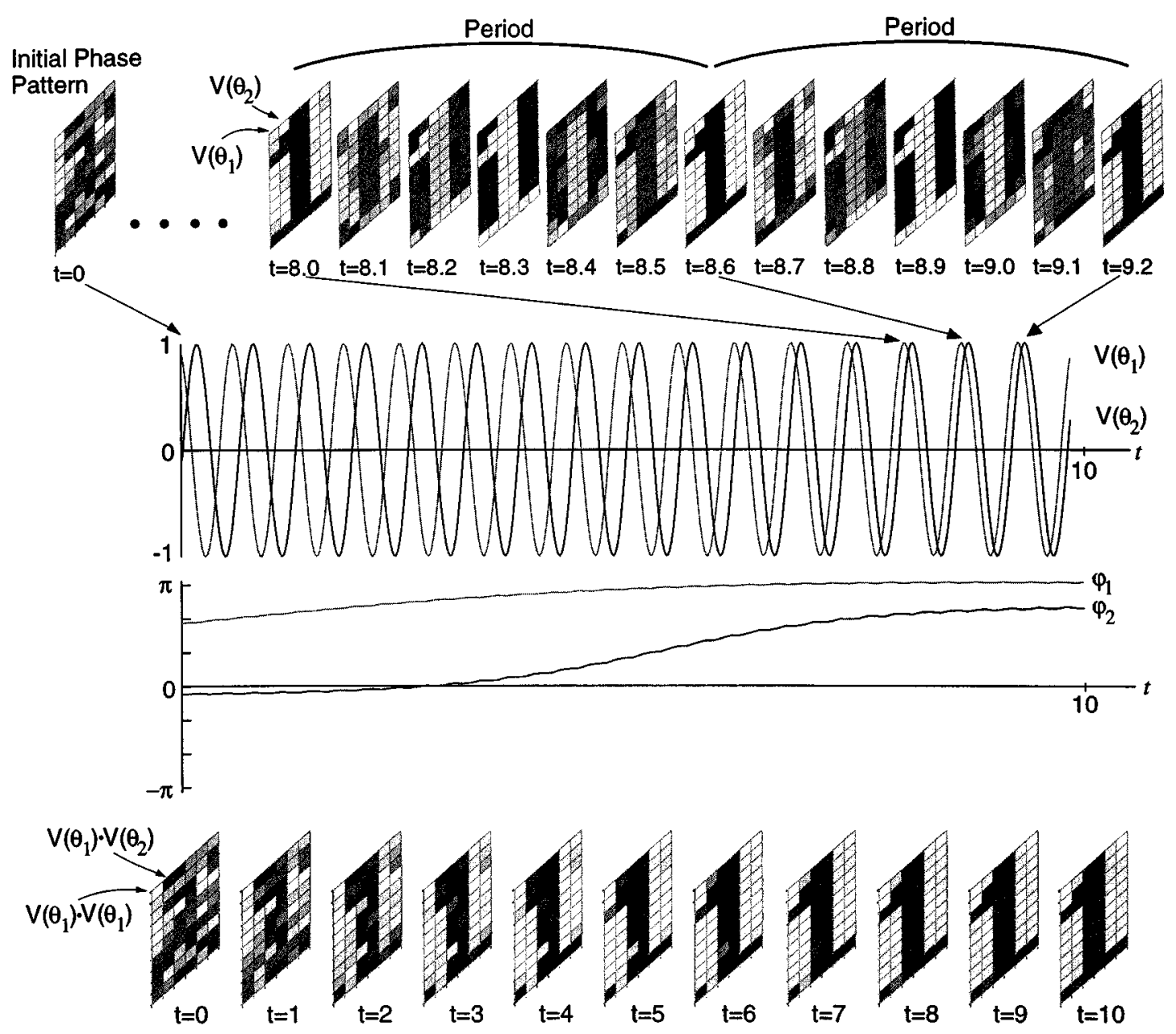

Fig. 5. Pattern recognition by the PLL neural network (1) with Hebbian learning rule (7) applied to the key patterns in Fig. 4. Functions and parameters: $V(\vartheta)=$ $\sin \vartheta, \Omega=10, n=60$, and $t \in[0,10]$.

The generalized PLL neural network (8) has more than one implementation. A straightforward one is when each circuit connecting two neurons has modifiable weight $s_{i j}$ and phase shift $\psi_{i j}$. However, if $V(\vartheta)=\sin \vartheta$, then we can represent (8) in an alternative form

$$
\dot{\vartheta}_{i}=\Omega+V\left(\vartheta_{i}\right) \sum_{j=1}^{n}\left(v_{i j} V\left(\vartheta_{j}-\pi / 2\right)+w_{i j} V\left(\vartheta_{j}\right)\right)
$$

where

$$
v_{i j}=\operatorname{Re} c_{i j}=s_{i j} \cos \psi_{i j}
$$

and

$$
w_{i j}=\operatorname{Im} c_{i j}=s_{i j} \sin \psi_{i j} .
$$

Using both $V\left(\vartheta_{j}\right)$ and $V\left(\vartheta_{j}-\pi / 2\right)$ to affect the $i$ th neuron eliminates the necessity for $n^{2}$ modifiable phase shifts, which simplifies the circuit.

\section{DISCUSSION}

We have proposed a novel architecture of an oscillatory neural network that can be built using off-the-shelf PLL's, e.g., LMC568 or LM565 series by National Semiconductor. The network can memorize and reproduce complex oscillatory patterns in which all neurons oscillate with the same frequency, but different phase relations. There are still some issues that have not been addressed.

- Oscillatory memory. What are the major advantages of oscillatory associative memory?

- Learning. What is the best learning rule for PLL neural networks in terms of the capacity? The Hebbian rule, which we used here, is the simplest one, but usually not the best.

- Waveforms. What are the advantages of various waveforms $V(\vartheta)$, e.g., those in Fig. 2.

- Scaling properties. How large can $n$ be, and what are the restrictions on the frequency $\Omega$ ?

- Acquisition time. How long does it take for the network to lock to the memorized pattern?

- Phase noise. How does the phase noise affect the PLL network performance?

Obviously, more research is needed before the PLL neural networks can pass the "small prototype" stage.

\section{REFERENCES}

[1] T. Aoyagi, "Network of neural oscillators for retrieving phase information," Phys. Rev. Lett., vol. 74, pp. 4075-4078, 1995. 
[2] B. Baird, "Nonlinear dynamics of pattern formation and pattern recognition in the rabbit olfactory bulb," Physica D, vol. 22, pp. 150-175, 1986.

[3] M. A. Cohen and S. Grossberg, "Absolute stability of global pattern formation and parallel memory storage by competitive neural networks," IEEE Trans. Syst., Man, Cybern., vol. SMC-13, pp. 815-826, 1983.

[4] C. M. Gray, "Synchronous oscillations in neuronal systems: Mechanism and functions," J. Computat. Neurosci., vol. 1, pp. 11-38, 1994.

[5] F. C. Hoppensteadt, "Intermittent chaos, self-organization, and learning from synchronous synaptic activity in model neuron networks," in Proc. National Academy of Sciences USA, vol. 86, 1989, pp. 2991-2995.

[6] - An Introduction to the Mathematics of Neurons. Modeling in the Frequency Domain. Cambridge, U.K.: Cambridge Univ. Press, 1997.

[7] F. C. Hoppensteadt and E. M. Izhikevich, "Oscillatory neurocomputers with dynamic connectivity," Phys. Rev. Lett., vol. 82, pp. 2983-2986, 1999.

[8] _ - "Thalamo-cortical interactions modeled by weakly connected oscillators: Could brain use FM radio principles?," BioSystems, vol. 48, pp. 85-94, 1998.

[9] - Weakly Connected Neural Networks. New York: SpringerVerlag, 1997

[10] - "Synaptic organizations and dynamical properties of weakly connected neural oscillators: I. Analysis of canonical model," Biol. Cybern., vol. 75, pp. 117-127, 1996.

[11] - "Synaptic organizations and dynamical properties of weakly connected neural oscillators. II. Learning of phase information," Biol. Cybern., vol. 75, pp. 129-135, 1996.

[12] P. Horowitz and W. Hill, The Art of Electronics, 2nd ed. Cambridge, U.K.: Cambridge Univ. Press, 1989.

[13] E. M. Izhikevich, "Resonate-and-fire neurons,", to be published.

[14] — ,Weakly connected quasiperiodic oscillators, FM interactions, and multiplexing in the brain," SIAM J. Appl. Math., vol. 59, pp. 2193-2223, 1999.

[15] — , "Weakly pulse-coupled oscillators, FM interactions, synchronization, and oscillatory associative memory," IEEE Trans. Neural Networks, vol. 10, pp. 508-526, 1999.
[16] Z. Li and J. J. Hopfield, "Modeling the olfactory bulb and its neural oscillatory processings," Biol. Cybern., vol. 61, pp. 379-392, 1989.

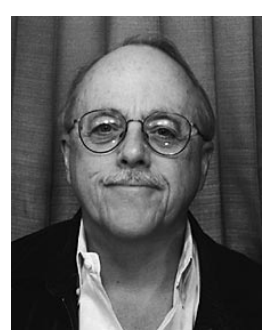

Frank C. Hoppensteadt (M'89) was born in Oak Park, IL, in 1938. He received the Ph.D. degree in mathematics from the University of Wisconsin, Madison.

He has published 12 books and numerous articles about perturbation methods for differential equations and mathematical problems in the life sciences. His present interests are in mathematical neuroscience, biotechnology, and random perturbations of dynamical systems. He is currently Director of the Regent's Center in System Science and Engineering Research, Professor of mathematics, and Professor of electrical engineering at Arizona State University, Tempe.

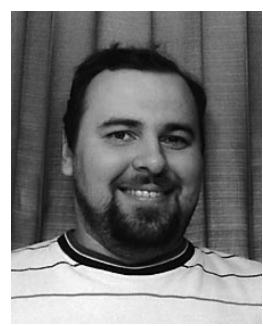

Eugene M. Izhikevich was born in Moscow, Russia, in 1967. He received the M.S. degree in applied mathematics and computer sciences from Lomonosov Moscow State University, Moscow, in 1992 and the Ph.D. degree in mathematics from Michigan State University, East Lansing, in 1996.

He is currently with the The Neurosciences Institute, La Jolla, CA. His is interested in nonlinear dynamical systems and bifurcations in neuron and brain dynamics.

Dr. Izhikevich received the SIAM Student Award for the best student paper in applied mathematics in 1995. He is a member of the International Neural Network Society. 